\title{
ARTICLE
}

\section{Potential role of TBC1D4 in enhanced post-exercise insulin action in human skeletal muscle}

\author{
J. T. Treebak • C. Frøsig • C. Pehmøller • S. Chen - S. J. Maarbjerg • N. Brandt • \\ C. MacKintosh • J. R. Zierath • D. G. Hardie • B. Kiens • E. A. Richter • H. Pilegaard • \\ J. F. P. Wojtaszewski
}

Received: 19 January 2009 / Accepted: 22 January 2009/Published online: 28 February 2009

(C) Springer-Verlag 2009

\begin{abstract}
Aims/hypothesis TBC1 domain family, member 4 (TBC1D4; also known as AS160) is a cellular signalling intermediate to glucose transport regulated by insulin-dependent and -independent mechanisms. Skeletal muscle insulin sensitivity is increased after acute exercise by an unknown mechanism that does not involve modulation at proximal insulin signalling intermediates. We hypothesised that signalling through TBC1D4 is involved in this effect of exercise as it is a common signalling element for insulin and exercise.
\end{abstract}

J. T. Treebak · C. Frøsig · C. Pehmøller · S. J. Maarbjerg $\cdot$

N. Brandt $\cdot$ B. Kiens $\cdot$ E. A. Richter $\cdot$ J. F. P. Wojtaszewski $(\bowtie)$

Copenhagen Muscle Research Centre, Molecular Physiology

Group, Department of Exercise and Sport Sciences,

University of Copenhagen,

DK-2100 Copenhagen, Denmark

e-mail: jwojtaszewski@ifi.ku.dk

S. Chen $\cdot$ C. MacKintosh

MRC Protein Phosphorylation Unit, College of Life Sciences,

University of Dundee,

Dundee, Scotland, UK

\section{J. R. Zierath}

Section for Integrative Physiology, Department of Molecular

Medicine and Surgery, Karolinska Institutet,

Stockholm, Sweden

D. G. Hardie

Division of Molecular Physiology, College of Life Sciences,

University of Dundee,

Dundee, Scotland, UK

\section{H. Pilegaard}

Copenhagen Muscle Research Centre and Centre of Inflammation and Metabolism, Department of Biology,

University of Copenhagen,

Copenhagen, Denmark
Methods Insulin-regulated glucose metabolism was evaluated in 12 healthy moderately trained young men $4 \mathrm{~h}$ after one-legged exercise at basal and during a euglycaemichyperinsulinaemic clamp. Vastus lateralis biopsies were taken before and immediately after the clamp.

Results Insulin stimulation increased glucose uptake in both legs, with greater effects $(\sim 80 \%, p<0.01)$ in the previously exercised leg. TBC1D4 phosphorylation, assessed using the phospho-AKT (protein kinase B) substrate antibody and phospho- and site-specific antibodies targeting six phosphorylation sites on TBC1D4, increased at similar degrees to insulin stimulation in the previously exercised and rested legs $(p<0.01)$. However, TBC1D4 phosphorylation on Ser-318, Ser-341, Ser-588 and Ser-751 was higher in the previously exercised leg, both in the absence and in the presence of insulin $(p<0.01$; Ser-588, $p=0.09$; observed power $=0.39)$. 14 $-3-3$ binding capacity for TBC1D4 increased equally $(p<0.01)$ in both legs during insulin stimulation.

Conclusion/interpretation We provide evidence for sitespecific phosphorylation of TBC1D4 in human skeletal muscle in response to physiological hyperinsulinaemia. The data support the idea that TBC1D4 is a nexus for insulin- and exercise-responsive signals that may mediate increased insulin action after exercise.

Keywords AKT substrate of $160 \mathrm{kDa} \cdot \mathrm{AS} 160$.

Glucose metabolism · TBC1D1

$\begin{array}{ll}\text { Abbreviations } \\ \text { AKT } & \text { Protein kinase B } \\ \text { AMPK } & \text { AMP-activated kinase } \\ \text { GAP } & \text { GTPase-activating proteins } \\ \text { PAS } & \text { Phospho-AKT substrate } \\ \text { PVDF } & \text { Polyvinylidene fluoride }\end{array}$

Abbreviations

AMPK AMP-activated kinase

GAP GTPase-activating proteins

PVDF Polyvinylidene fluoride 
TBC1D1 TBC1 domain family, member 1

TBC1D4 TBC1 domain family, member 4

\section{Introduction}

Insulin sensitivity of skeletal muscle to stimulate glucose uptake is markedly enhanced by a prior bout of exercise. This effect is restricted to the previously contracted muscle, rather than a consequence of changes in systemic factors [1-8]. The enhanced insulin sensitivity that increases glucose transport after acute exercise is associated with recruitment of GLUT4 molecules to the plasma membrane [9], but not with GLUT4 protein levels [10]. This effect cannot be linked to increased signal transduction at the insulin receptor or intermediates in proximal components of the insulin signalling cascade involving IRS1, phosphoinositide 3-kinase, AKT (protein kinase B) and glycogen synthase kinase $3[8,10,11]$.

TBC1 domain family, member 1 (TBC1D1) and member 4 (TBC1D4) are GTPase-activating proteins (GAP) and direct targets of AKT $[12,13]$. They are similar in size and structure and run at approximately 150 to $160 \mathrm{kDa}$ during SDS-PAGE [14]. The GAP domain of both TBC1D1 and TBC1D4 stimulates the GTPase activity of certain Rab proteins, thereby inhibiting GLUT4 translocation to the plasma membrane $[13,15,16]$. GTPase activity is thought to be regulated by phosphorylation and both TBC1D1 and TBC1D4 proteins contain multiple Ser/Thr residues that can be phosphorylated by various kinases [13, 14, 17]. In line with this, stimulation by aminoimidazole carboxamide ribonucleotide, insulin and other growth factors, as well as contraction of skeletal muscle, leads to phosphorylation of TBC1D1 and/or TBC1D4 [18-22]. Beside phosphorylation, binding of 14-3-3 proteins to phosphorylated Thr-642 and/or Ser-341 of TBC1D4 [18, 23] is important for glucose transport in 3T3-L1 adipocytes possibly via regulation of the GAP domain [23].

Being multi-kinase substrates, TBC1D1 and TBC1D4 may be a point of convergence for insulin-dependent and insulin-independent signalling pathways regulating glucose transport in skeletal muscle. Indeed, both insulin- and contraction-induced glucose uptake in skeletal muscle have been shown to partly depend on TBC1D4 [24]. Consequently, the intracellular signals emanating from the contracting muscle during exercise may interact with the insulin signalling pathway at the TBC1D1 or TBC1D4 level, thereby mediating enhanced glucose uptake in previously exercised muscle [25]. Recent studies of rodent and human skeletal muscle provide evidence for a role of TBC1D4 in this respect [26-28], showing that TBC1D4 phosphorylation is increased in the hours following an acute bout of exercise. However, although one study in rat skeletal muscle has shown that prior exercise resulted in enhanced basal and insulin-stimulated glucose uptake and phosphorylation of TBC1D4 [26], no such evidence has been generated in humans.

Phosphorylation of TBC1D1 and TBC1D4 has previously been evaluated using the phospho-AKT substrate (PAS) antibody, which recognises phosphorylation sites on AKT substrates lying in a $(\mathrm{R} / \mathrm{K}) \mathrm{X}(\mathrm{R} / \mathrm{K}) \mathrm{XXS} * \mathrm{~T}^{*}$ sequence motif. Thus, the interpretation of previous investigations has been limited by the unselective nature of the PAS antibody, which may recognise multiple phosphorylation sites on TBC1D1 and TBC1D4 and mask potential sitespecific effects of exercise or insulin. It was recently shown, however, that only Thr-642 on TBC1D4 and Thr596 on TBC1D1 are recognised by the PAS antibody [13, 18, 19], leaving several phosphorylation sites that are potentially important for GAP activity unrecognised. Furthermore, since TBC1D1 and TBC1D4 have similar mobility on SDS-PAGE and since both proteins are recognised by the PAS antibody, data from previous investigations using this antibody should be interpreted carefully $[12,21,22,29-35]$. To circumvent these problems, we used phospho-specific antibodies against six phosphorylation sites on TBC1D4. Our findings suggest that site-specific TBC1D4 phosphorylation events may play a role in mediating the beneficial effects of exercise on skeletal muscle insulin action.

\section{Methods}

Participants The participants in this study were 12 young, healthy men (age $24 \pm 1$ years; weight $80 \pm 2.7 \mathrm{~kg}$; height $188 \pm 2 \mathrm{~cm}$; BMI $23 \pm 0.5 \mathrm{~kg} / \mathrm{m}^{2} ; \mathrm{VO}_{2 \text { peak }} 4.6 \pm 0.1 \mathrm{l} / \mathrm{min}$ ), who gave informed consent to participate. The study was approved by the local Ethics Committee in the municipality of Copenhagen (01-180/01) and performed in accordance with the Helsinki declaration.

Experimental design Following 4 days of standardised diet (65\% of energy as carbohydrate, $20 \%$ as fat, $15 \%$ as protein) the participants arrived at the laboratory in the morning after an overnight fast. They ate a small, carbohydrate-rich snack ( $5 \%$ daily energy intake) and then performed $60 \mathrm{~min}$ of dynamic one-legged knee extensor exercise (one kick/s) at $~ 80 \%$ of peak workload of the knee extensors [8]. During the $60 \mathrm{~min}$ of exercise, two $5 \mathrm{~min}$ phases at $100 \%$ of peak workload were performed to ensure activation of the majority of the vastus lateralis muscle. The contralateral leg was kept at rest. After exercise, participants rested in the supine position for $4 \mathrm{~h}$. During the resting period participants had Teflon catheters inserted below the inguinal ligament in one femoral artery and both 
femoral veins and after $4 \mathrm{~h}$ of rest a 100 min euglycaemic-

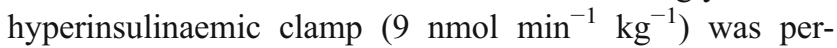
formed as described previously [11]. Before and immediately after the clamp, biopsies from the vastus lateralis muscle were taken under subcutaneous anaesthesia $(\sim 2-$ $3 \mathrm{ml}$ xylocain [10 mg/ml lidocaine]; AstraZeneca, Södertälje, Sweden) from the rested and exercised leg using individual incisions. Specifically, to address mRNA isoform expression of $T B C 1 D 1$ and $T B C 1 D 4$, biopsies were obtained from abdominal subcutaneous adipose tissue and vastus lateralis muscle from eight participants in the rested, non-stimulated state. All biopsies were quickly $(<20 \mathrm{~s})$ frozen in liquid nitrogen and stored at $-80^{\circ} \mathrm{C}$.

Blood chemistry Glucose levels during the clamp were measured in whole blood using an ABL 615 (Radiometer Medical, Brønshøj, Denmark). Plasma insulin concentrations were determined by RIA (DSL-1600; Diagnostic Systems Laboratories, Webster, TX, USA).

Muscle and adipose tissue processing Muscle tissue was freeze-dried, dissected free of visible fat and connective tissue. Muscle and adipose tissue samples were homogenised in ice-cold buffer $(50 \mathrm{mmol} / \mathrm{l} \mathrm{HEPES}, 150 \mathrm{mmol} / \mathrm{l} \mathrm{NaCl}$, $20 \mathrm{mmol} / 1 \quad \mathrm{Na}_{4} \mathrm{P}_{2} \mathrm{O}_{7}, 20 \mathrm{mmol} / \mathrm{l} \quad \beta$-glycerophosphate, $10 \mathrm{mmol} / \mathrm{l} \mathrm{NaF}, 2 \mathrm{mmol} / 1 \mathrm{Na}_{3} \mathrm{VO}_{4}, 2 \mathrm{mmol} / \mathrm{l}$ EDTA, $1 \%$ (vol./vol.) Nonidet P-40, 10\% (vol./vol.) glycerol, $2 \mathrm{mmol} / \mathrm{l}$ phenylmethylsulfonyl fluoride, $10 \mu \mathrm{g} / \mathrm{ml}$ leupeptin, $10 \mu \mathrm{g} / \mathrm{ml}$ aprotinin and $3 \mathrm{mmol} / \mathrm{l}$ benzamidine). Homogenates were rotated end-over-end at $4^{\circ} \mathrm{C}$ for $1 \mathrm{~h}$ and subjected to centrifugation $\left(17,500 \times g, 4^{\circ} \mathrm{C}\right)$ for $20 \mathrm{~min}$. Supernatant fractions were collected and protein concentrations determined (Pierce, Rockford, IL, USA).

Glycogen Muscle glycogen was determined as glucosyl units after acid hydrolysis. The analysis was performed by fluorometry using freeze dried, dissected muscle specimens as described [36].

Antibodies Phosphorylation of TBC1D1 and TBC1D4 was measured using the PAS antibody (Cell Signaling Technology, Boston, MA, USA) and phospho-specific antibodies against TBC1D4 (Ser-318, Ser-341, Ser-588, Thr-642, Ser666 and Ser-751) as previously described [18]. Total TBC1D4 protein was determined using an antibody specific for human TBC1D4 (Abcam, Cambridge, UK). For 14-3-3 overlays, an antibody made against the C-terminal part of TBC1D4 (KAKIGNKP) was applied in the immunoprecipitation step as described below. Total TBC1D1 antibody was generated as described previously [19]. We have previously determined that the TBC1D4 total antibody from Abcam and the PAS antibody specifically detect TBC1D4 in human skeletal muscle $[37,38]$. Specificity of the TBC1D4 phospho-specific antibodies and the TBC1D1 antibody has been confirmed using various cell lines [18, 19]. To further confirm specificity of the TBC1D4 phospho-specific antibodies, we performed immunodepletion analyses using TBC1D1 and TBC1D4 antibodies and muscle lysates from human skeletal muscle.

Immunoprecipitation of TBC1D4 and TBC1D1 TBC1D4 and TBC1D1 were immunoprecipitated from $300 \mu \mathrm{g}$ of protein from human skeletal muscle lysates using antibodies bound to protein $G$ agarose beads as previously described [37]. Supernatant fractions from samples were removed and immunocomplexes were subsequently washed two times in PBS. The immunocomplexes were boiled in Laemmli buffer and subjected to SDS-PAGE probing for TBC1D4 and TBC1D1 protein and TBC1D4 phosphorylation using the antibodies described above.

SDS-PAGE and Western blot analyses Western blot analyses were performed as previously described [37]. Membranes probed with the phospho-specific antibodies for TBC1D4 were stripped in a buffer containing $100 \mathrm{mmol} / \mathrm{l}$ 2 -mercaptoethanol, $0.02 \mathrm{~g} / \mathrm{ml} \mathrm{SDS}$ and $62.5 \mathrm{mmol} / 1$ Tris- $\mathrm{HCl}$ (pH 6.7), and re-probed with TBC1D4 antibody. The signal from the phospho-specific antibodies was related to total TBC1D4 in the sample.

Phosphorylation of Ser-666 Phosphorylation of Ser-666 on TBC1D4 was measured by immunoprecipitating TBC1D4 protein from $150 \mu \mathrm{g}$ of human skeletal muscle lysate. The precipitant was subjected to SDS-PAGE as described above and after visualisation membranes were stripped and normalised to TBC1D4 protein as described above.

14-3-3 overlays We did 14-3-3 overlays as previously described [39]. Briefly, endogenous TBC1D4 protein was immunoprecipitated from $150 \mu \mathrm{g}$ of human muscle lysates, electrophoretically separated on gels (Nu-PAGE; Invitrogen, Paisley, UK) and subsequently transferred on to nitrocellulose membranes. Membranes were probed overnight at $4{ }^{\circ} \mathrm{C}$ with digoxigenin-labelled 14-3-3, followed by incubation with a horseradish-peroxidase-conjugated anti-digoxigenin antibody (Roche, Burgess Hill, UK). Immune complexes were visualised and quantified (Odyssey Infrared Imaging System; LI-COR Biosciences, Lincoln, NE, USA), and normalised to TBC1D4 protein.

$R N A$ isolation, reverse transcription, $P C R$ and calculations Procedures for isolation of RNA, reverse transcription, determination of single-stranded cDNA content and PCR have been described previously [40]. Primers and probes used for the PCR reaction are presented in Table 1. All TaqMan probes were 5'-6-carboxyfluorescein- and 3'-6- 
Table 1 Oligo sequences for primers and probes used for amplifying human TBC1D1, TBC1D4-short and TBC1D4-long mRNA

\begin{tabular}{ll}
\hline Gene & Forward primer \\
TBC1D1 & 5'-GTGTGGGAAAAGATGCTTAGCA-3' \\
TBC1D4-short & 5'-AGCTCCAGTGAACAGTGCAGTG-3' \\
TBC1D4-long & 5'-GAGGCAGAGCTCCAGTGAACA 3' \\
& Reverse primer \\
TBC1D1 & 5'-GTGATGACGTGGCACACCTT-3' \\
TBC1D4-short & 5'-CACTTAGGGACTCATTGCTGC 3' \\
TBC1D4-long & 5'-GACTTGGAAGACTGGAGGAAGAATT 3' \\
& TaqMan probe \\
TBC1D1 & 5'-ACATGGAAAAAATGCACTCGGCTGTTG-3' \\
TBC1D4-short & 5'-ATGGAGAAGGGAGAAAAAGGACCTCATCTAC 3' \\
TBC1D4-long & 5'-CAATCTTTCGTCAGTTCGACGCATGTACAA 3' \\
\hline
\end{tabular}

carboxy- $N, N, N^{\prime}, N^{\prime}$-tetramethylrhodamine-labelled. Individual standard curves were generated for each gene using a serial dilution of a pooled skeletal muscle and a pooled adipose tissue cDNA sample. Based on the slopes of the standard curves, amplification efficiencies were similar for the three sets of primers $(91.6 \%, 88.7 \%$ and $91.2 \%$ for $T B C 1 D 1, T B C 1 D 4$-short form and TBC1D4-long form, respectively). Furthermore, the mRNA expression level of the different genes was calculated by linear regression $(y=\alpha x+\beta)$ using the gene-specific slopes (amplification efficiencies) of the standard curves and a common $\beta$ value. The mRNA content of each gene was normalised to total single-stranded cDNA in the specific sample.

Statistics All statistical analyses were conducted using SPSS 16.0.1 (SPSS, Chicago, IL, USA). Levene's test of equality of variance was used to confirm that all groups had equal variances. Two-way analyses of variance with repeated measures for the factors 'leg' and 'time' were used to test for main effects and interactions. A $p$ value of $p<0.05$ was considered statistically significant. Data are expressed as means $\pm \mathrm{SE}$.

\section{Results}

Skeletal muscle glycogen and whole-body glucose uptake At $4 \mathrm{~h}$ after exercise, skeletal muscle glycogen was lower in the previously exercised leg $(397 \pm 61 \mu \mathrm{mol} / \mathrm{g})$ than in the rested leg $(618 \pm 48 \mu \mathrm{mol} / \mathrm{g})$. Insulin levels reached a plateau of $680 \pm 5 \mathrm{pmol} / \mathrm{l}$ after $15 \mathrm{~min}$ of insulin stimulation. Thigh glucose uptake was similar before the clamp commenced, but was significantly higher $(p<0.01)$ in the previously exercised leg $(6.7 \pm 0.6 \mathrm{mmol} / \mathrm{kg}$ [AUC] $)$ than in the rested leg during insulin stimulation $(3.9 \pm 0.6 \mathrm{mmol} / \mathrm{kg}[\mathrm{AUC}])$, indicating insulin action was enhanced in response to prior exercise. This study is part of a larger investigation, which is designed to assess metabolism and signalling events in skeletal muscle in the post-exercise state. Additional data from the experiment described here are presented in a previous publication [11].

Phosphorylation of TBC1D4 after exercise Using the PAS antibody and site-specific antibodies against TBC1D4, we determined the phosphorylation status of TBC1D4 $4 \mathrm{~h}$ after termination of an acute exercise bout, just before and at the end of a euglycaemic-hyperinsulinaemic clamp. TBC1D4 phosphorylation level determined using the PAS antibody in response to insulin was substantially increased $(p<0.01)$, but to a similar level whether the legs had previously been rested or exercised (Fig. 1a). This phosphorylation pattern coincided with phosphorylation of Thr-642 and Ser-666 (Fig. 1b, c), which was also increased in response to insulin $(p<0.01$ for main effect), but was unaltered between the rested and exercised leg. Phosphorylation of all other sites on TBC1D4 (Ser-318, Ser-341, Ser-588, Ser-751) measured in these samples followed the same pattern: insulin induced a marked phosphorylation in both the previously rested and previously exercised legs $(p<0.01$ for main effect of insulin). In addition, prior exercise was associated with increased ( $p<0.01$ for main effect) phosphorylation compared with the previously rested leg, regardless of effects of insulin treatment on these four sites (Fig. 1d-g). The main effect of prior exercise in the Ser-588 data was only of borderline significance $(p=0.09$; observed power $=0.39)$ (Fig. 1f).

14-3-3 binding capacity of TBC1D4 It has been shown that 14-3-3 binds TBC1D4 in response to insulin treatment of cultured cells. To evaluate whether 14-3-3 also binds to TBC1D4 in skeletal muscle in response to insulin and whether the binding capacity was altered by prior exercise, we performed 14-3-3 overlay assays as previously described [18]. The increase in binding capacity of 14-3-3 to TBC1D4 in the previously exercised and rested leg in response to insulin was similar. At $4 \mathrm{~h}$ after exercise, binding capacity of 14-3-3 to TBC1D4 was not increased in the non-stimulated muscle (Fig. 2).

Test of TBC1D1, TBC1D4 and phospho-TBC1D4 antibody specificity To test the specificity of total TBC1D4 and TBC1D1 antibodies, we immunodepleted TBC1D4 and TBC1D1 from human skeletal muscle lysates and performed Western blot analyses (Fig. 3a-c). Both antibodies were able to completely remove all TBC1D4 or TBC1D1 protein from the supernatant fractions. However, a very faint band detected by the TBC1D4 antibody was seen in the TBC1D1 immunoprecipitate (Fig. 3a). Furthermore, 
Fig. 1 TBC1D4 phosphorylation in the post-exercise state. TBC1D4 phosphorylation was evaluated in vastus lateralis muscle samples obtained in the basal state $4 \mathrm{~h}$ after termination of a one-legged knee extensor exercise bout (white bars) and at the end of a euglycaemichyperinsulinaemic clamp (black bars) using the PAS antibody and phospho-specific antibodies targeting six of the known phosphorylation sites on TBC1D4. Values, in arbitrary units (AU), are for (a) PAS, (b) Thr-642, (c) Ser-666, (d) Ser318, (e) Ser-341, (f) Ser-588 and (g) Ser-751. $* * p<0.01$ for insulin effect, $n=12 ;{ }^{\dagger \dagger} p<0.01$ for prior exercise effect, $n=12$; ${ }^{(\dagger)} p=0.09$ borderline significance, observed power $=0.39$, $n=12$. h Representative blots using TBC1D4 phosphospecific antibodies. All antibodies gave a single band running at $160 \mathrm{kDa}$. Ser-666 was measured after immunoprecipitation of TBC1D4; all other blots were direct blots performed using vastus lateralis muscle lysates
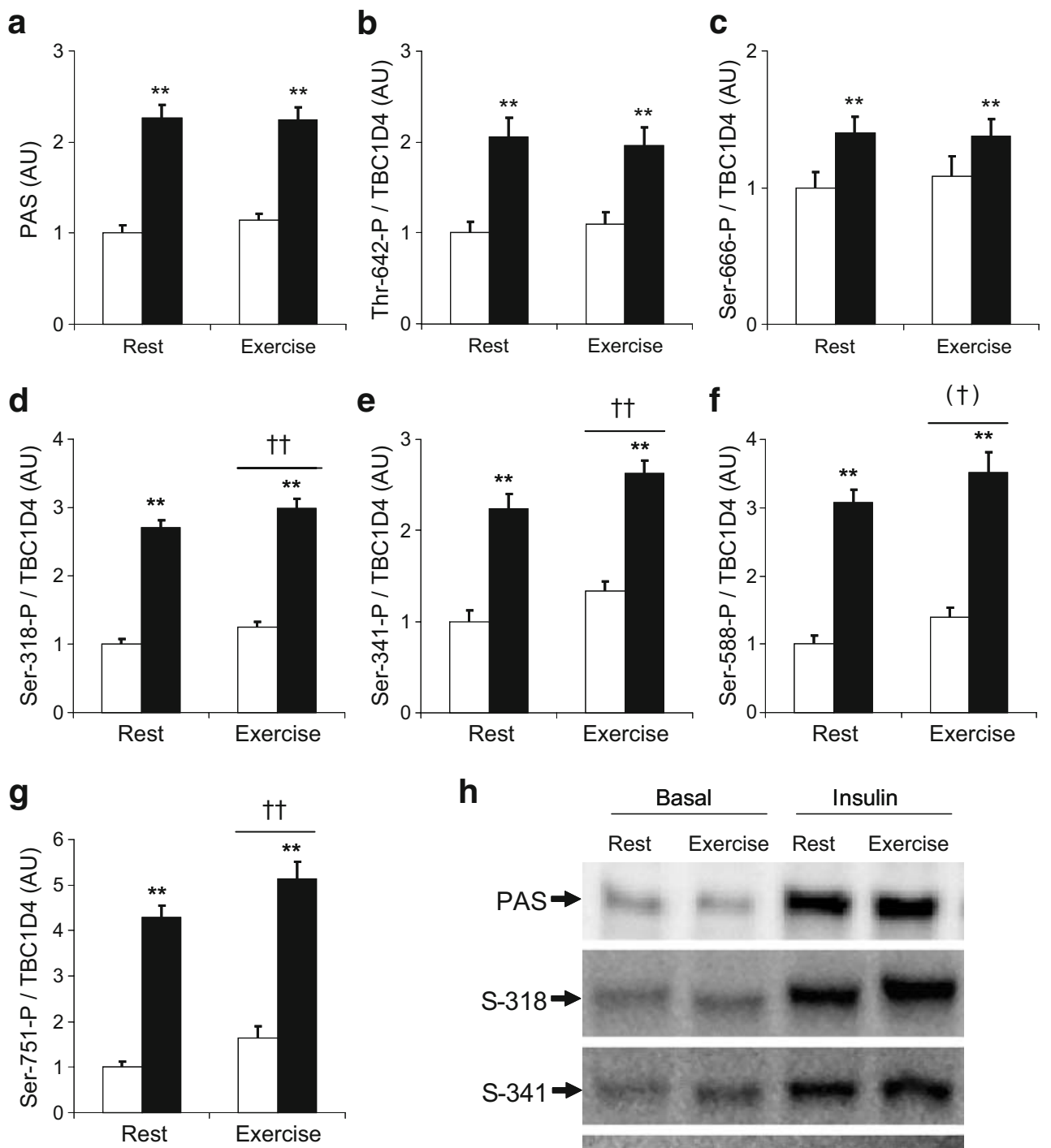

when TBC1D4 was immunoprecipitated and the pellet blotted for TBC1D1, we detected a clear band running at $150 \mathrm{kDa}$ (Fig. 3b). Thus, TBC1D4 can be co-immunoprecipitated with TBC1D1 and vice versa. By re-probing the membrane shown in Fig. 3a with a mix of TBC1D1 and TBC1D4 antibodies, it became clear that TBC1D1 and TBC1D4 run with different molecular mass (Fig. 3c). Thus, we conclude that TBC1D1 and TBC1D4 antibodies do not cross-react when used for direct blotting. In mouse skeletal muscle, TBC1D1 is highly expressed and the signal obtained with

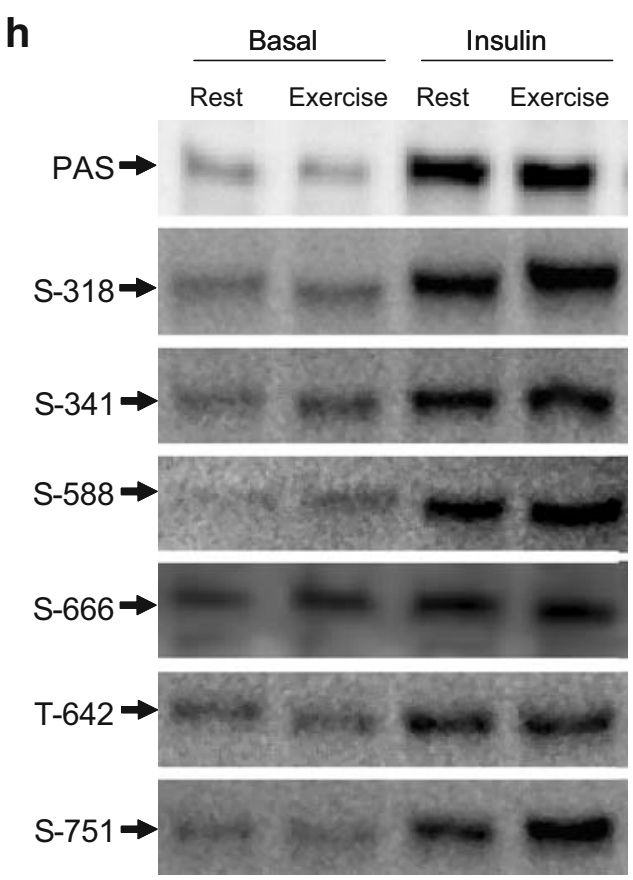

the PAS antibody associates primarily with TBC1D1 [14]. To evaluate this issue in human skeletal muscle, we performed split blots by cutting the membrane through one lane and blotting for TBC1D4 and/or TBC1D1 and/or PAS (Fig. 3d). Clearly, the signal obtained with the PAS antibody followed TBC1D4 and not TBC1D1 in human skeletal muscle. These data are consistent with our observations that immunoprecipitation of TBC1D4 completely depletes the PAS signal from human skeletal muscle obtained in the nonstimulated state [37, 38], both during insulin stimulation 


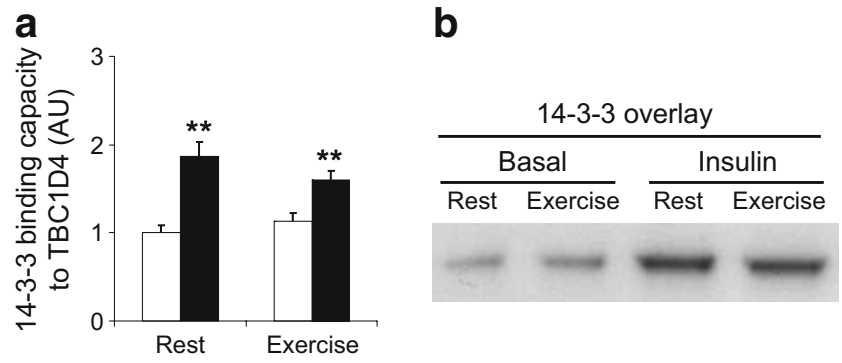

Fig. 2 Binding capacity of 14-3-3 to TBC1D4 increased with insulin as shown in (a). Following immunoprecipitation of TBC1D4, 14-3-3 binding capacity to TBC1D4 was evaluated by far-western overlay (b) using digoxigenin-labelled $14-3-3$ protein. ${ }^{* *} p<0.01$ for insulin effect, $n=12$

(Fig. 3, [38]) and following exercise [37]. Thus, under various conditions detectable PAS signal originated from TBC1D4 in human skeletal muscle. Finally, to show that the TBC1D4 phospho-specific antibodies detect only TBC1D4 and not TBC1D1 in human skeletal muscle, we immunoprecipitated TBC1D4 and incubated with different phospho-TBC1D4 antibodies (Fig. 3e). All TBC1D4 phospho-antibodies detected a single band running at the same molecular mass or slightly higher than the lysate sample. Furthermore, no bands are detected in the superna- tant fractions. Thus, based on the results presented in Fig. 3, together with previously published data [18], we conclude that the phospho-antibodies specifically detect TBC1D4 in human skeletal muscle.

mRNA expression of TBC1D1 and TBC1D4 isoforms in human vastus lateralis muscle and subcutaneous adipose tissue Transcripts from two splice variants of mouse $T B C 1 D 4$ and TBC1D1 exist in the databases [13]. Furthermore, it has been found that skeletal muscle and white adipose tissue from mice differentially express the different splice variants [14]. It is not known whether these differences are also present in human skeletal muscle and subcutaneous abdominal adipose tissue. From the transcript sequences available, specific primers and probes were generated directed against the short and long splice variants of $T B C 1 D 4$. Additionally and since only the short splice variant of $T B C 1 D 1$ is available in the databases, one set of primers that amplify both the short and long variants of $T B C 1 D 1$ was generated. Our results showed that subcutaneous adipose tissue from humans expressed TBC1D1 mRNA and only the short version of TBC1D4, and levels of TBC1D1 mRNA were approximately threefold greater than those of TBC1D4 mRNA (Fig. 4a). Skeletal muscle a

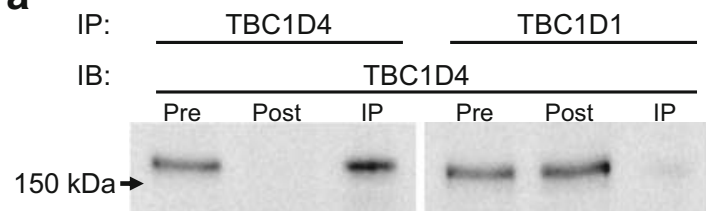

b

IP:

TBC1D4

TBC1D1

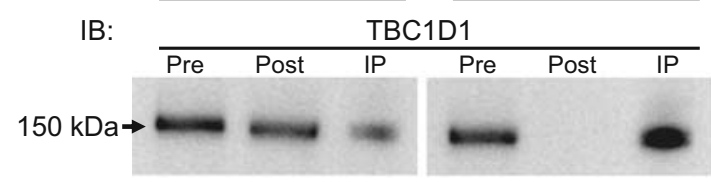

C

IP:

TBC1D4

TBC1D1

IB:

TBC1D4+TBC1D1

$150 \mathrm{kD}$

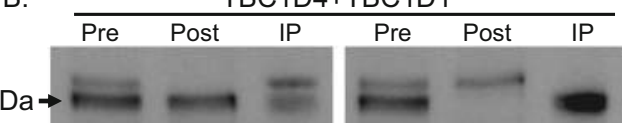

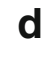

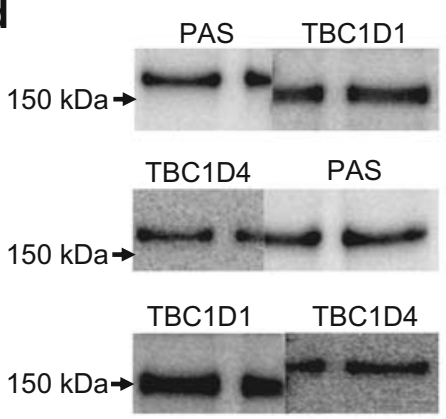

e
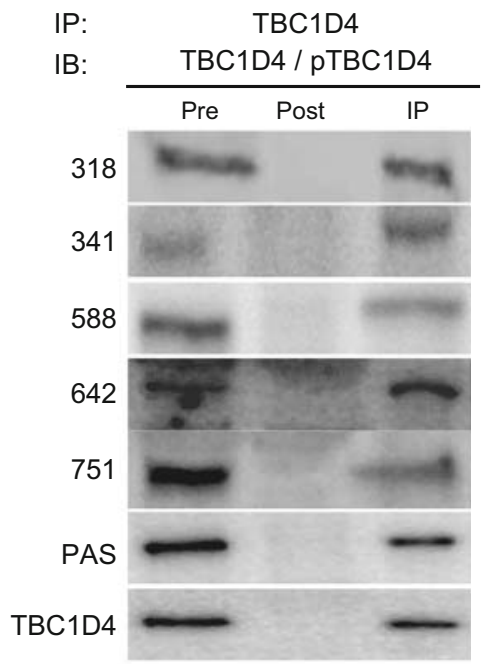

Fig. 3 Verification of antibodies specificity. a TBC1D1 and TBC1D4 were immunoprecipitated from a human vastus lateralis muscle sample and the immunoprecipitates (IP) $(150 \mu \mathrm{g})$ loaded together with the pre-IPs (lysate cleared with beads; $30 \mu \mathrm{g}$ ) and the post-IPs (supernatant fraction; $30 \mu \mathrm{g}$ ). Following SDS-PAGE proteins were transferred to PVDF membranes, which were then probed with TBC1D4 antibody. b Same samples as above (a), but the membranes were incubated with TBC1D1 antibody. c Membranes (a) were reincubated with a mixture of TBC1D1 and TBC1D4 antibodies and developed to show the separation between TBC1D1 and TBC1D4. d Western blot analyses were performed using an insulin-stimulated human vastus lateralis muscle sample loaded in three wells. The membrane was cut through the middle lane. The two membranes were incubated with different antibodies as indicated. The different pieces of membrane were put back together and developed. TBC1D1 and TBC1D4 ran at different molecular masses, whereas the signal from the PAS antibody aligned with TBC1D4, but not with TBC1D1. e TBC1D4 was immunoprecipitated from the same sample used above (d), and pre-IP $(60 \mu \mathrm{g})$, post-IP $(60 \mu \mathrm{g})$ and IP $(120 \mu \mathrm{g})$ were subjected to SDS-PAGE and transferred to PVDF membranes. These were then incubated with antibodies as indicated. All bands in the IP lane ran with the same, or slightly higher, molecular mass as the preIP sample. IB, immunoblot 
a

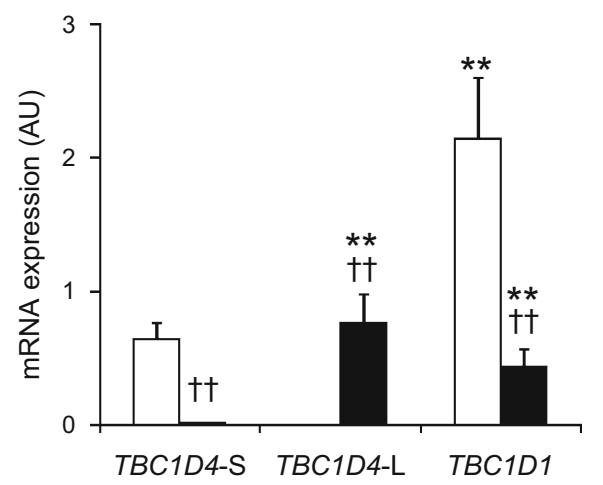

b

IB: TBC1D4

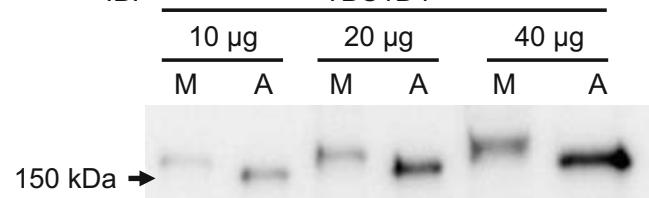

Fig. 4 a mRNA expression of human TBC1D1 and TBC1D4 splice variants in skeletal muscle and subcutaneous adipose tissue. Relative amounts of the different gene products were calculated and compared. Adipose tissue (white bars) exclusively expressed the short (-S) version of TBC1D4 and TBC1D1. Expression of TBC1D1 was approximately threefold higher than that of TBC1D4 in adipose tissue. Skeletal muscle (black bars) expressed equal amounts of TBC1D4long (-L) and $T B C 1 D 1$, but only very small amounts of $T B C 1 D 4-\mathrm{S}$, which may be contamination from inter-myocellular fat cells. b TBC1D4 Western blot showing that human skeletal muscle (M) and subcutaneous adipose tissue (A) differentially produce TBC1D4-L and TBC1D4-S splice variant proteins. ${ }^{* *} p<0.01$ vs TBC1D4-S within tissue, $(n=8) ;{ }^{\dagger \dagger} p<0.01$ for within gene, $n=8$. IB, immunoblot

expressed very small amounts of TBC1D4-short mRNA, which could have been contamination from fat cells lying between the muscle fibres. Moreover, TBC1D4-long and $T B C 1 D 1$ mRNA were approximately equally abundant in human skeletal muscle. Using an antibody that detects both splice variants of TBC1D4, we confirmed that TBC1D4short and TBC1D4-long mRNA are exclusively associated with subcutaneous adipose tissue and skeletal muscle, respectively (Fig. 4b). Within the amount of protein loaded on the gel, we detected only a single band in each sample running with the molecular mass corresponding to TBC1D4-short and TBC1D4-long. Thus, these data confirm our mRNA data and are in agreement with data obtained from mouse tissues $[13,14]$.

\section{Discussion}

We investigated whether acute exercise influences basal and insulin-stimulated TBC1D4 phosphorylation in skeletal muscle $4 \mathrm{~h}$ after an acute bout of exercise. We used phospho-specific antibodies directed against six phosphorylation sites on TBC1D4 to determine whether TBC1D4 was phosphorylated in the post-exercise state when insulin action was increased in the previously exercised leg. We show that TBC1D4 phosphorylation is increased in response to physiological insulin stimulation on all of the six sites investigated. Moreover, phosphorylation of Ser-318, Ser-341, Ser-588 and Ser-751 was increased by prior exercise, both before and immediately after insulin stimulation. The differential phosphorylation of TBC1D4 is a product of TBC1D4 kinases and phosphatases regulated during and after exercise. Thus, it is likely that exercise directly induces TBC1D4 phosphorylation on Ser-318, Ser341, Ser-588 and Ser-751. However, we cannot rule out the possibility that Thr-642 and Ser-666 are also phosphorylated during exercise, but due to faster time-courses for dephosphorylation no changes are present at $4 \mathrm{~h}$ after exercise. Regardless of this, TBC1D4 phosphorylation was maintained on specific sites in the hours after exercise, so when insulin is present, translocation and fusion of GLUT4 molecules to the plasma membrane may be potentiated in the exercised muscle. Therefore, TBC1D4 may be a 'point of convergence' between insulin- and exercise-mediated signalling pathways $[25,41]$. Activation of AMP-activated protein kinase (AMPK) in exercising human skeletal muscle is associated with PAS-phosphorylation of TBC1D4 [37], and in rodents, AMPK is involved in the phosphorylation of TBC1D1 and/or TBC1D4 during contraction [21, 22]. So although various regulators may be involved, it is tempting to speculate that AMPK is one kinase involved in the observed regulation of TBC1D4.

TBC1D4 regulation in human skeletal muscle has primarily been assessed using the PAS antibody, which recognises phosphorylated Ser/Thr residues in a $(\mathrm{R} / \mathrm{K}) \mathrm{X}(\mathrm{R} / \mathrm{K}) \mathrm{XXS}^{*} / \mathrm{T}^{*}$ motif sequence. Although most of the known TBC1D4 phosphorylation sites have this lead-up sequence, antibody recognition cannot be guaranteed. In fact, data indicate that Thr-642 may be the primary phosphorylation site recognised by the PAS antibody [18]. Interestingly, of all the phosphorylation sites in the perfect consensus sequence for detection by the PAS antibody (Thr-642, Ser-318, Ser-341, Ser-588 and Ser-751,) only Thr-642 showed a pattern of phosphorylation similar to that detected using the PAS antibody.

Little is known about TBC1D4 regulation in skeletal muscle in the post-exercise state. In healthy humans, TBC1D1 and/or TBC1D4 phosphorylation assessed using the PAS antibody was enhanced after endurance type exercise, whereas the response to insulin was not [27]. Likewise, in the hours after a bout of resistance exercise, PAS phosphorylation was also elevated in human skeletal muscle [28]. In contrast, we show that while PAS phosphorylation is 
unchanged, TBC1D4 phosphorylation at specific sites (Ser318, Ser-341 and Ser-751) is enhanced both in the basal and insulin-stimulated state $4 \mathrm{~h}$ after exercise. Time, mode and intensity of the exercise bout, and the following resting period may explain the differences between this study and previously published data $[27,28]$. A likely scenario is that exercise as such increases PAS phosphorylation [31,37] and when the exercise is terminated, PAS phosphorylation gradually decreases towards baseline. Thus, in the present study, we cannot exclude the possibility that basal PAS phosphorylation was still increased $\sim 3 \mathrm{~h}$ into recovery but reached baseline at $4 \mathrm{~h}$. Moreover, the enhanced basal and insulin-stimulated PAS phosphorylation observed in rat skeletal muscle after exercise [26] was not observed here in human skeletal muscle. While all available data point to a role for TBC1D4 in the post-exercise situation, regulation of TBC1D4 may differ across exercise regimes and animal species.

In this study we also investigated the ability of 14-3-3 proteins to interact with TBC1D4. We provide evidence that 14-3-3 binds to TBC1D4 in response to insulin, confirming previous findings [27]. Cell studies suggest that 14-3-3 binds to TBC1D4 Ser-341 and Thr-642 upon phosphorylation $[18,23]$. It is possible that other sites on TBC1D4 bind 14-3-3 in vivo, although this may be difficult to prove due to weak association between TBC1D4 and 14-3-3 [18]. Nevertheless, as 14-3-3 binding was not elevated by prior exercise (Fig. 2a), this binding may not be important in the enhanced insulin action observed. Also, the present data would suggest that Ser-318, Ser-341 and Ser751 do not contribute to $14-3-3$ binding, since the phosphorylation pattern and 14-3-3 binding do not coincide. Statistical analyses revealed that the interaction effect in the 14-3-3 data was of borderline significance $(p=0.08$; observed power= $0.54)$. Thus, we may have missed a real effect due to lack of statistical power. However, if prior exercise does decrease the ability of insulin to promote 14-3-3 binding, this would be counterintuitive given previous data suggesting 14-3-3 binding promotes inhibition of TBC1D4 and thus increases GLUT4 translocation [23].

TBC1D4 shares similar features to TBC1D1 and is also present in mouse skeletal muscle [13, 14, 42]. The GAP domain of the two proteins is highly similar and both proteins are thought to be regulated by phosphorylation. Thus, TBC1D4 and TBC1D1 may play redundant roles in tissues in which they are present. There is now strong evidence to support a role for TBC1D1 and TBC1D4 in regulating glucose transport in skeletal muscle and adipocytes $[13,17,24,43]$. Thus, TBC1D1 may also be important for the increased insulin sensitivity in skeletal muscle after exercise. The sequence around Thr-642 in TBC1D4 is similar to the sequence around Thr-596 in TBC1D1, and data from 3T3-L1 adipocytes and HEK-293 cells indicate that Thr-596 is the main phosphorylation site detected by the PAS antibody [13, 19]. TBC1D1 is abundant in mouse skeletal muscle [14, 42], and TBC1D1 and TBC1D4 migrate at almost the same molecular mass during SDSPAGE [14]. In addition, phosphorylation measured by the PAS antibody in mouse skeletal muscle containing a high proportion of type II glycolytic fibres actually originates from TBC1D1 [14]. This raises the question of whether both TBC1D4 and TBC1D1 phosphorylation were determined in our previous studies in human skeletal muscle, where the PAS antibody was used for immunoblot experiments [37, 38, 44]. In fact, TBC1D1 is expressed in human skeletal muscle at the mRNA level (Fig. 4) and at the protein level (Fig. 3). Still, we verified our previous finding showing PAS phosphorylation in response to insulin can be completely accounted for in TBC1D4 immunoprecipitates $[37,38]$. Also, TBC1D1 and TBC1D4 migrated with visibly different molecular mass (Fig. 3d) and the detectable PAS signal was exclusively associated with TBC1D4 and not with TBC1D1 in human skeletal muscle. We cannot easily explain the observed differences between human and mouse skeletal muscle regarding the different levels of TBC1D1 and TBC1D4, and the level of the PAS signal associated with these proteins. However, mouse skeletal muscles are generally highly glycolytic [45] compared with human vastus lateralis muscle [46], so levels of TBC1D1 and TBC1D4 are likely to be associated with the myosin heavy-chain isoform expression profile.

We showed in Fig. 3a, b that TBC1D1 was partially pulled down in the TBC1D4 immunoprecipitate and viceversa. We cannot exclude the possibility that the TBC1D1 and TBC1D4 antibodies detect TBC1D1 and TBC1D4 in their native, folded conformation. However, it is more likely that TBC1D1 and TBC1D4 dimerise, so when TBC1D4 is immunoprecipitated TBC1D1 co-precipitates [19]. Although the quantitative level of these proteins is unknown, the present finding indicates that the fraction of total TBC1D1 associated with TBC1D4 is larger than the fraction of total TBC1D4 that associates with TBC1D1. To further explore the relation between TBC1D1 and TBC1D4, we measured the mRNA content of TBC1D1 and $T B C 1 D 4$ splice variants, both in human skeletal muscle and subcutaneous adipose tissue. While skeletal muscle primarily expressed TBC1D4-long and TBC1D1 in equal amounts, adipose tissue contained more $T B C 1 D 1$ than TBC1D4-short. This mRNA expression profile is in contrast to protein content previously reported in mouse skeletal muscle and white adipose tissue [14, 42]. Thus, further investigation is necessary to determine whether our mRNA data translate directly into protein content in human skeletal muscle and white adipose tissue.

In summary, we have used phospho-specific antibodies to provide direct evidence for insulin-induced phosphor- 
ylation of TBC1D4. Moreover, phosphorylation of Ser-318, Ser-341, Ser-588 $(p=0.09$; observed power $=0.39)$ and Ser751 was increased in the basal, as well as insulin-stimulated state $4 \mathrm{~h}$ after acute exercise. This finding constitutes an advance in our understanding of the molecular mechanisms underlying enhanced skeletal muscle insulin action after exercise. Furthermore, we provide new insight into the potential mechanism by which insulin- and exercisemediated signalling pathways converge on TBC1D4 to increase glucose uptake in skeletal muscle.

Acknowledgements We would like to thank I. Bech Nielsen and B. Bolmgren for their skilled technical assistance during the experiments. Financial support was from the Copenhagen Muscle Research Centre, the Danish Natural Science Research Council, the Danish Medical Research Council, the Novo Nordisk Foundation, the Danish Diabetes Association, the Lundbeck Foundation, the Swedish Research Council and from an integrated project (contract number LSHM-CT-2004005272) from the European Union. J. F. P. Wojtaszewski was supported by a Hallas Møller fellowship from the Novo Nordisk Foundation.

Duality of interest The authors declare that there is no duality of interest associated with this manuscript.

\section{References}

1. Richter EA, Garetto LP, Goodman MN, Ruderman NB (1982) Muscle glucose metabolism following exercise in the rat: increased sensitivity to insulin. J Clin Invest 69:785-793

2. Bogardus C, Thuillez P, Ravussin E, Vasquez B, Narimiga M, Azhar S (1983) Effect of muscle glycogen depletion on in vivo insulin action in man. J Clin Invest 72:1605-1610

3. Garetto LP, Richter EA, Goodman MN, Ruderman NB (1984) Enhanced muscle glucose metabolism after exercise in the rat: the two phases. Am J Physiol Endocrinol Metab 246:E471-E475

4. Richter EA, Garetto LP, Goodman MN, Ruderman NB (1984) Enhanced muscle glucose metabolism after exercise: modulation by local factors. Am J Physiol Endocrinol Metab 246:E476-E482

5. Mikines KJ, Sonne B, Farrell PA, Tronier B, Galbo H (1988) Effect of physical exercise on sensitivity and responsiveness to insulin in humans. Am J Physiol Endocrinol Metab 254:E248E259

6. Cartee GD, Young DA, Sleeper MD, Zierath J, WallbergHenriksson H, Holloszy JO (1989) Prolonged increase in insulin-stimulated glucose transport in muscle after exercise. Am J Physiol Endocrinol Metab 256:E494-E499

7. Richter EA, Mikines KJ, Galbo H, Kiens B (1989) Effect of exercise on insulin action in human skeletal muscle. J Appl Physiol 66:876-885

8. Wojtaszewski JF, Hansen BF, Kiens B, Richter EA (1997) Insulin signaling in human skeletal muscle: time course and effect of exercise. Diabetes 46:1775-1781

9. Hansen PA, Nolte LA, Chen MM, Holloszy JO (1998) Increased GLUT-4 translocation mediates enhanced insulin sensitivity of muscle glucose transport after exercise. J Appl Physiol 85:12181222

10. Wojtaszewski JFP, Hansen BF, Gade J et al (2000) Insulin signaling and insulin sensitivity after exercise in human skeletal muscle. Diabetes 49:325-331
11. Frøsig C, Sajan MP, Maarbjerg SJ et al (2007) Acute exercise improves PIP3 responsiveness of aPKC and interacts with insulin signalling to peptide elongation in human skeletal muscle. J Physiol (Lond) 582:1289-1301

12. Kane S, Sano H, Liu SCH et al (2002) A method to identify serine kinase substrates. Akt phosphorylates a novel adipocyte protein with a Rab GTPase-activating protein (GAP) domain. J Biol Chem 277:22115-22118

13. Roach WG, Chavez JA, Miinea CP, Lienhard GE (2007) Substrate specificity and effect on GLUT4 translocation of the Rab GTPaseactivating protein Tbc1d1. Biochem J 403:353-358

14. Taylor EB, An D, Kramer HF et al (2008) Discovery of TBC1D1 as an Insulin-, AICAR-, and contraction-stimulated signaling nexus in mouse skeletal muscle. J Biol Chem 283:9787-9796

15. Miinea CP, Sano H, Kane S et al (2005) AS160, the Akt substrate regulating GLUT4 translocation, has a functional Rab GTPase activating protein domain. Biochem J 391:87-93

16. Larance M, Ramm G, Stockli J et al (2005) Characterization of the role of the Rab GTPase-activating protein AS160 in insulinregulated GLUT4 trafficking. J Biol Chem 280:37803-37813

17. Sano H, Kane S, Sano E et al (2003) Insulin-stimulated phosphorylation of a Rab GTPase-activating protein regulates GLUT4 translocation. J Biol Chem 278:14599-14602

18. Geraghty K, Chen S, Harthill JE et al (2007) Regulation of multisite phosphorylation and 14-3-3 binding of AS160 in response to insulin-like growth factor 1, EGF, PMA and AICAR. Biochem J 407:231-241

19. Chen S, Murphy J, Toth R, Campbell DG, Morrice NA, MacKintosh C (2008) Complementary regulation of TBC1D1 and AS160 by growth factors, insulin and AMPK activators. Biochem J 409:449-459

20. Thong FSL, Bilan PJ, Klip A (2007) The Rab GTPase-activating protein AS160 integrates Akt, protein kinase C, and AMPactivated protein kinase signals regulating GLUT4 Traffic. Diabetes 56:414-423

21. Treebak JT, Glund S, Deshmukh A et al (2006) AMPK-mediated AS160 phosphorylation in skeletal muscle is dependent on AMPK catalytic and regulatory subunits. Diabetes 55:2051-2058

22. Kramer HF, Witczak CA, Fujii N et al (2006) Distinct signals regulate AS160 phosphorylation in response to insulin, AICAR, and contraction in mouse skeletal muscle. Diabetes 55:2067-2076

23. Ramm G, Larance M, Guilhaus M, James DE (2006) A role for 14-3-3 in insulin-stimulated GLUT4 translocation through its interaction with the RabGAP AS160. J Biol Chem 281:2917429180

24. Kramer HF, Witczak CA, Taylor EB, Fujii N, Hirshman MF, Goodyear LJ (2006) AS160 regulates insulin- and contractionstimulated glucose uptake in mouse skeletal muscle. J Biol Chem 281:31478-31485

25. Cartee GD, Wojtaszewski JF (2007) Role of Akt substrate of 160 $\mathrm{kDa}$ in insulin-stimulated and contraction-stimulated glucose transport. Appl Physiol Nutr Metab 32:557-566

26. Arias EB, Kim J, Funai K, Cartee GD (2007) Prior exercise increases phosphorylation of Akt substrate of $160 \mathrm{kDa}$ (AS160) in rat skeletal muscle. Am J Physiol Endocrinol Metab 292:E1191E1200

27. Howlett K, Mathews A, Garnham A, Sakamoto K (2008) The effect of exercise and insulin on AS160 phosphorylation and 143-3 binding capacity in human skeletal muscle. Am J Physiol Endocrinol Metab 294:E401-E407

28. Dreyer HC, Drummond MJ, Glynn EL, et al (2008) Resistance exercise increases human skeletal muscle AS160/TBC1D4 phosphorylation in association with enhanced leg glucose uptake during post-exercise recovery. J Appl Physiol 105:1967-1974

29. Bruss MD, Arias EB, Lienhard GE, Cartee GD (2005) Increased phosphorylation of Akt substrate of $160 \mathrm{kDa}$ (AS160) in rat 
skeletal muscle in response to insulin or contractile activity. Diabetes 54:41-50

30. Bouzakri K, Karlsson HKR, Vestergaard H, Madsbad S, Christiansen E, Zierath JR (2006) IRS-1 Serine phosphorylation and insulin resistance in skeletal muscle from pancreas transplant recipients. Diabetes 55:785-791

31. Deshmukh A, Coffey VG, Zhong Z, Chibalin AV, Hawley JA, Zierath JR (2006) Exercise-induced phosphorylation of the novel Akt substrates AS160 and filamin A in human skeletal muscle. Diabetes 55:1776-1782

32. Karlsson HK, Zierath JR, Kane S, Krook A, Lienhard GE, Wallberg-Henriksson H (2005) Insulin-stimulated phosphorylation of the Akt substrate AS160 is impaired in skeletal muscle of type 2 diabetic subjects. Diabetes 54:1692-1697

33. Karlsson HK, Hallsten K, Bjornholm M et al (2005) Effects of metformin and rosiglitazone treatment on insulin signaling and glucose uptake in patients with newly diagnosed type 2 diabetes: a randomized controlled study. Diabetes 54:1459-1467

34. Karlsson HKR, Ahlsen M, Zierath JR, Wallberg-Henriksson H, Koistinen HA (2006) Insulin signaling and glucose transport in skeletal muscle from first-degree relatives of type 2 diabetic patients. Diabetes 55:1283-1288

35. Peck GR, Ye S, Pham V et al (2006) Interaction of the Akt substrate, AS160, with the glucose transporter 4 vesicle marker protein, insulin-regulated aminopeptidase. Mol Endocrinol 20:2576-2583

36. Passonneau JV, Gatfield PD, Schulz DW, Lowry OH (1967) An enzymic method for measurement of glycogen. Anal Biochem 19:315-326

37. Treebak JT, Birk JB, Rose AJ, Kiens B, Richter EA, Wojtaszewski JF (2007) AS160 phosphorylation is associated with activation of $\alpha 2 \beta 2 \gamma 1$ but not $\alpha 2 \beta 2 \gamma 3$ AMPK trimeric complex in skeletal muscle during exercise in humans. Am J Physiol Endocrinol Metab 292:E715-E722
38. Højlund K, Glintborg D, Andersen NR et al (2008) Impaired insulin-stimulated phosphorylation of Akt and AS160 in skeletal muscle of women with polycystic ovary syndrome is reversed by pioglitazone treatment. Diabetes 57:357-366

39. Pozuelo Rubio M, Geraghty KM, Wong BHC et al (2004) 14-33 -affinity purification of over 200 human phosphoproteins reveals new links to regulation of cellular metabolism, proliferation and trafficking. Biochem J 379:395-408

40. Lundby C, Hellsten Y, Jensen MBF, Munch AS, Pilegaard H (2008) Erythropoietin receptor in human skeletal muscle and the effects of acute and long-term injections with recombinant human erythropoietin on the skeletal muscle. J Appl Physiol 104:1154-1160

41. Treebak JT, Wojtaszewski JF (2008) Role of 5'AMP-activated protein kinase in skeletal muscle. Int J Obes (Lond) 32(Suppl 4): S13-S17

42. Chavez JA, Roach WG, Keller SR, Lane WS, Lienhard GE (2008) Inhibition of GLUT4 translocation by Tbc1d1, a Rab GTPaseactivating protein abundant in skeletal muscle, is partially relieved by AMP-activated protein kinase activation. J Biol Chem 283:9187-9195

43. Chadt A, Leicht K, Deshmukh A et al (2008) Tbc1d1 mutation in lean mouse strain confers leanness and protects from diet-induced obesity. Nat Genet 40:1354-1359

44. Frøsig C, Rose AJ, Treebak JT, Kiens B, Richter EA, Wojtaszewski JFP (2007) Effects of endurance exercise training on insulin signaling in human skeletal muscle: interactions at the level of phosphatidylinositol 3-kinase, Akt, and AS160. Diabetes 56:2093-2102

45. Allen DL, Harrison BC, Sartorius C, Byrnes WC, Leinwand LA (2001) Mutation of the IIB myosin heavy chain gene results in muscle fiber loss and compensatory hypertrophy. Am J Physiol Cell Physiol 280:C637-C645

46. Pilegaard H, Terzis G, Halestrap A, Juel C (1999) Distribution of the lactate $/ \mathrm{H}+$ transporter isoforms MCT1 and MCT4 in human skeletal muscle. Am J Physiol Endocrinol Metab 276:E843-E848 